\title{
Schwarz-Pick inequalities for the Schur-Agler class on the polydisk and unit ball
}

\author{
J. Milne Anderson, Michael A. Dritschel, and James Rovnyak \\ To Walter Hayman on his 80-th birthday
}

\begin{abstract}
The notion of a unitary realization is used to estimate derivatives of arbitrary order of functions in the Schur-Agler class on the polydisk and unit ball.
\end{abstract}

Keywords. Schwarz-Pick inequality, realization, transfer function, SchurAgler class, Arveson space.

2000 MSC. 30C80 (Primary); 32A30, 47B32, 47A48 (Secondary).

\section{Introduction}

The classical Schwarz-Pick estimate is the inequality

$$
\left|\varphi^{\prime}(z)\right| \leq \frac{1-|\varphi(z)|^{2}}{1-|z|^{2}}, \quad|z|<1,
$$

for an analytic function $\varphi(z)$ which is bounded by one on the unit disk of the complex plane. Ruscheweyh [24] has obtained best-possible estimates of higher order derivatives of bounded analytic functions on the disk. Similar estimates were derived by other methods and for different classes of analytic functions in one and several variables by Anderson and Rovnyak [5], Avkhadiev and Wirths [7, 8, 9], Bénéteau, Dahlner, and Khavinson [13], and MacCluer, Stroethoff, and Zhao [21, 22]. In this paper we derive estimates of derivatives of functions on the polydisk and unit ball using realizations of functions as transfer functions of systems or, equivalently, as characteristic functions of operator colligations.

The notion of a realization plays a key role in many areas of operator theory, linear systems theory, and interpolation problems, where the class of analytic functions which are bounded by one on the unit disk $\mathbb{D}=\{z:|z|<1\}$ is often called the Schur class. By a realization of an analytic function $\varphi(z)$ in the Schur class on $\mathbb{D}$ is meant a representation in the form

$$
\varphi(z)=D+z C\left(I_{\mathcal{H}}-z A\right)^{-1} B, \quad z \in \mathbb{D},
$$

J. Milne Anderson acknowledges the support of the Leverhulme Trust. 
where $A, B, C, D$ are the entries of a block operator matrix

$$
U=\left(\begin{array}{ll}
A & B \\
C & D
\end{array}\right): \underset{\mathbb{C}}{\stackrel{\mathcal{H}}{\oplus}} \rightarrow \underset{\mathbb{C}}{\oplus}
$$

for some Hilbert space $\mathcal{H}$. Such a representation always exists and can be chosen such that $U$ is a unitary operator on $\mathcal{H} \oplus \mathbb{C}$ (for example, see Brodskiu [15, Theorem 5.1]). In this case (11) is called a unitary realization of $\varphi(z)$. It turns out that the estimates of Ruscheweyh 24] have straightforward proofs by means of (1), and moreover this method extends to several variables.

The notion of a unitary realization can be introduced for a broad class of domains $\Omega$ in $\mathbb{C}^{d}$. The Schur class on $\Omega$ is the set of analytic functions which are defined and bounded by one on $\Omega$. A unitary realization of a function $\varphi(z)$ in the Schur class on $\Omega$ is a representation

$$
\varphi(z)=D+C Z(z)\left(I_{\mathcal{K}}-A Z(z)\right)^{-1} B, \quad z \in \Omega,
$$

where

$$
U=\left(\begin{array}{ll}
A & B \\
C & D
\end{array}\right): \underset{\mathbb{C}}{\stackrel{\mathcal{H}}{\oplus}} \rightarrow \underset{\mathbb{C}}{\bigoplus}
$$

is a unitary operator for some Hilbert spaces $\mathcal{H}$ and $\mathcal{K}$, and $Z(z)$ is a function of $z=\left(z_{1}, \ldots, z_{d}\right)$ in $\Omega$ with values in $\mathcal{L}(\mathcal{K}, \mathcal{H})$ such that

(i) $\|Z(z)\|<1$ for all $z \in \Omega$.

For the domains that we consider, we also assume:

(ii) $Z\left(z_{1}, \ldots, z_{d}\right)=z_{1} E_{1}+\cdots+z_{d} E_{d}$ where $E_{j} \in \mathcal{L}(\mathcal{K}, \mathcal{H})$ and $\left\|E_{j}\right\| \leq 1$, $j=1, \ldots, d$.

Condition (i) assures that the inverse in (3) exists for each $z \in \Omega$. Condition (ii) is assumed for convenience: it is satisfied in our applications, and it simplifies differentiation formulas because higher order derivatives of the linear function $Z(z)$ vanish. The estimates that we derive below generalize to nonlinear functions $Z(z)$, but the nonlinear case is more complicated because the formulas for higher order derivatives involve additional terms. As this generality is not needed here, we only treat the linear case.

Unitary realizations of the form (3) include (1) as a special case when $d=1$ and $\Omega=\mathbb{D}$. We describe the situation for the polydisk $\mathbb{D}^{d}=\mathbb{D} \times \cdots \times \mathbb{D}$ and unit ball $\mathbb{B}_{d}=\left\{\left(z_{1}, \ldots, z_{d}\right):\left|z_{1}\right|^{2}+\cdots+\left|z_{d}\right|^{2}<1\right\}$ in greater detail.

1.1. The Schur-Agler class on the polydisk. Let $\Omega=\mathbb{D}^{d}$. A complexvalued analytic function $\varphi(z)$ on $\mathbb{D}^{d}$ is said to belong to the Schur-Agler class on the polydisk if for every set $T_{1}, \ldots, T_{d}$ of commuting contractions on a Hilbert space and every positive number $r<1,\left\|\varphi\left(r T_{1}, \ldots, r T_{d}\right)\right\| \leq 1$. For $d=1$ and $d=2$, the Schur-Agler class coincides with the Schur class on $\mathbb{D}^{d}$. For $d>2$, the Schur-Agler class is a proper subset of the Schur class on $\mathbb{D}^{d}$ (for example, see [20] and [26]). For any $d \geq 1$, the class of functions which have a 
unitary realization (3) coincides with the Schur-Agler class on $\mathbb{D}^{d}$ when suitable choices are made. Namely, in (3) and (44) we take $\mathcal{K}=\mathcal{H}$ and assume that

$$
\mathcal{H}=\mathcal{H}_{1} \oplus \cdots \oplus \mathcal{H}_{d}
$$

for some closed subspaces $\mathcal{H}_{1}, \ldots, \mathcal{H}_{d}$ of $\mathcal{H}$. We also assume that

$$
Z\left(z_{1}, \ldots, z_{d}\right)=z_{1} E_{1}+\cdots+z_{d} E_{d}
$$

where $E_{1}, \ldots, E_{d}$ are the orthogonal projections of $\mathcal{H}$ onto $\mathcal{H}_{1}, \ldots, \mathcal{H}_{d}$. Alternatively, we can write the elements $h$ of $\mathcal{H}$ in column form:

$$
h=\left(\begin{array}{c}
h_{1} \\
\vdots \\
h_{d}
\end{array}\right) \text {. }
$$

Then $A, B, C$ can correspondingly be written in block operator form, and in this notation,

$$
Z\left(z_{1}, \ldots, z_{d}\right)=\left(\begin{array}{cccc}
z_{1} I_{\mathcal{H}_{1}} & 0 & \cdots & 0 \\
0 & z_{2} I_{\mathcal{H}_{2}} & \cdots & 0 \\
& & \cdots & \\
0 & 0 & \cdots & z_{d} I_{\mathcal{H}_{d}}
\end{array}\right) .
$$

With these conventions, every function of the form (3) belongs to the Schur-Agler class on $\mathbb{D}^{d}$, and conversely every function in the Schur-Agler class on $\mathbb{D}^{d}$ has this form. See Agler [1] and Ball and Trent [11].

Remark. The Schur-Agler class occurs naturally in the function theory of the polydisk. It is known that for every function $\varphi(z)$ in the Schur class on $\mathbb{D}^{d}$,

$$
\left(1-\left|z_{1}\right|^{2}\right)\left|\frac{\partial \varphi}{\partial z_{1}}\right|+\cdots+\left(1-\left|z_{d}\right|^{2}\right)\left|\frac{\partial \varphi}{\partial z_{d}}\right| \leq 1-|\varphi(z)|^{2}
$$

at every point $z=\left(z_{1}, \ldots, z_{d}\right)$ of $\mathbb{D}^{d}$ (see Rudin [23, p. 179] and Knese [18]). The cases of equality are determined by Knese [18]. All extremal functions belong to the Schur-Agler class. A Schur function $\varphi(z)$ on $\mathbb{D}^{d}$, which has no identically vanishing first partials, satisfies

$$
\left(1-\left|z_{1}\right|^{2}\right)\left|\frac{\partial \varphi}{\partial z_{1}}\right|+\cdots+\left(1-\left|z_{d}\right|^{2}\right)\left|\frac{\partial \varphi}{\partial z_{d}}\right|=1-|\varphi(z)|^{2}
$$

at every point of $\mathbb{D}^{d}$ if and only if it has the form (3), where $U$ is given by (4) with $\mathcal{H}=\mathcal{K}=\mathbb{C}^{d}$ and, in addition, $U$ is symmetric, that is, $U$ is equal to its transpose.

1.2. The Schur-Agler class on the unit ball. Let $\Omega=\mathbb{B}_{d}$. Set $\langle z, w\rangle=$ $z_{1} \bar{w}_{1}+\cdots+z_{d} \bar{w}_{d}$ for any $z=\left(z_{1}, \ldots, z_{d}\right)$ and $w=\left(w_{1}, \ldots, w_{d}\right)$ in $\mathbb{C}^{d}$, and write

$$
k(w, z)=\frac{1}{1-\langle z, w\rangle}, \quad z, w \in \mathbb{B}_{d} .
$$

The Arveson space is the Hilbert space $\mathcal{H}(k)$ of analytic functions on $\mathbb{B}_{d}$ with reproducing kernel $k(w, z)$ (for example, see Arveson [6] and Drury [16]). An analytic function $\varphi(z)$ on $\mathbb{B}_{d}$ is a multiplier of the Arveson space if multiplication 
by $\varphi(z)$ is an everywhere defined and bounded operator on $\mathcal{H}(k)$. It is called a contractive multiplier if this operator is a contraction, that is, it has norm at most one. Equivalently, $\varphi(z)$ is a contractive multiplier of the Arveson space if and only if the kernel $[1-\varphi(z) \overline{\varphi(w)}] /(1-\langle z, w\rangle)$ is nonnegative, that is, the inequality

$$
\sum_{j, k=1}^{n} \frac{1-\varphi\left(z^{(k)}\right) \overline{\varphi\left(z^{(j)}\right)}}{1-\left\langle z^{(k)}, z^{(j)}\right\rangle} c_{j} \bar{c}_{k} \geq 0
$$

holds for any points $z^{(1)}, \ldots, z^{(n)}$ in $\mathbb{B}_{d}$, any complex numbers $c_{1}, \ldots, c_{n}$, and any positive integer $n$. For $d=1$, the class of contractive multipliers of the Arveson space coincides with the Schur class on $\mathbb{B}_{d}$. For $d>1$, the set of contractive multipliers of the Arveson space is a proper subset of the Schur class (see [2, pp. 99-100] and [3, p. 6]). For any $d \geq 1$, the set of contractive multipliers of the Arveson space coincides with the class of functions which have a unitary realization (3) when suitable choices are made. In (44) we choose $\mathcal{K}=\mathcal{H} \oplus \cdots \oplus \mathcal{H}$ with $d$ summands and take

$$
Z\left(z_{1}, \ldots, z_{d}\right)=z_{1} E_{1}+\cdots z_{d} E_{d}
$$

where $E_{j}\left(h_{1} \oplus \cdots \oplus h_{d}\right)=h_{j}, j=1, \ldots, d$. Alternatively, write the elements of $\mathcal{K}$ in column form, and let

$$
A=\left(\begin{array}{c}
A_{1} \\
\vdots \\
A_{d}
\end{array}\right), \quad B=\left(\begin{array}{c}
B_{1} \\
\vdots \\
B_{d}
\end{array}\right) .
$$

Then (3) becomes

$$
\begin{aligned}
\varphi(z)=D+C Z(z)\left(I_{\mathcal{K}}-A Z(z)\right)^{-1} B= & D+C\left(I_{\mathcal{H}}-Z(z) A\right)^{-1} Z(z) B \\
& =D+C\left(I_{\mathcal{H}}-\sum_{j=1}^{d} z_{j} A_{j}\right)^{-1} \sum_{j=1}^{d} z_{j} B_{j} .
\end{aligned}
$$

With these choices, every function of the form (3) is a contractive multiplier of the Arveson space, and every contractive multiplier of the Arveson space has this form. For this reason, the class of contractive multipliers of the Arveson space is also called the Schur-Agler class on the unit ball. See Ball, Trent, and Vinnikov 12 and Eschmeier and Putinar [17.

In $\$ 2$ of this paper, we show how to estimate the expression (3) for functions on an arbitrary domain $\Omega$ in $\mathbb{C}^{d}$. Since no extra labor is involved, the results in $\$ 2$ are proved for operator-valued functions. Estimates for derivatives of complexvalued functions in the Schur-Agler class on the polydisk and unit ball are then deduced in $\$ 3$ and $\$ 4$ by interpreting the general results in $\$ 2$ for these cases. In $\S 3$ and $\$ 4$ we also derive special results for the polydisk and unit ball with the aid of new Hilbert space estimates, which appear in Lemmas 8 and 11. Extensions to operator-valued functions and some open questions are discussed in $\S 5$. 


\section{Estimates of functions which admit a realization}

Throughout this section we assume that $\varphi(z)$ is a function of $z=\left(z_{1}, \ldots, z_{d}\right)$ on a domain $\Omega$ in $\mathbb{C}^{d}$ with values in $\mathcal{L}(\mathcal{F}, \mathcal{G})$ for some Hilbert spaces $\mathcal{F}$ and $\mathcal{G}$, and that $\varphi(z)$ admits a representation in the form

$$
\varphi(z)=D+C Z(z)\left(I_{\mathcal{K}}-A Z(z)\right)^{-1} B, \quad z \in \Omega,
$$

where

$$
U=\left(\begin{array}{ll}
A & B \\
C & D
\end{array}\right): \underset{\mathcal{F}}{\stackrel{\mathcal{H}}{\oplus}} \rightarrow \underset{\mathcal{K}}{\oplus}
$$

is a unitary operator for some Hilbert spaces $\mathcal{H}$ and $\mathcal{K}$, and $Z(z)$ is a function of $z=\left(z_{1}, \ldots, z_{d}\right)$ in $\Omega$ with values in $\mathcal{L}(\mathcal{K}, \mathcal{H})$ such that

$$
\|Z(z)\|<1, \quad z \in \Omega .
$$

We also use the condition

$$
Z\left(z_{1}, \ldots, z_{d}\right)=z_{1} E_{1}+\cdots+z_{d} E_{d} \quad \text { where } \quad E_{j} \in \mathcal{L}(\mathcal{K}, \mathcal{H}), j=1, \ldots, d .
$$

The symbol $I$ always denotes an identity operator, and a subscript, when present, indicates the underlying space.

Lemma 1. Let $\varphi(z)$ have the form (8) where (9) is a unitary operator and $Z(z)$ satisfies (10). For all $w, z \in \Omega$,

$$
\begin{aligned}
I_{\mathcal{F}}-\varphi(z)^{*} & \varphi(w) \\
& =B^{*}\left(I_{\mathcal{K}}-Z(z)^{*} A^{*}\right)^{-1}\left(I_{\mathcal{K}}-Z(z)^{*} Z(w)\right)\left(I_{\mathcal{K}}-A Z(w)\right)^{-1} B \\
I_{\mathcal{G}}-\varphi(w) & \varphi(z)^{*} \\
& =C\left(I_{\mathcal{H}}-Z(w) A\right)^{-1}\left(I_{\mathcal{H}}-Z(w) Z(z)^{*}\right)\left(I_{\mathcal{H}}-A^{*} Z(z)^{*}\right)^{-1} C^{*} .
\end{aligned}
$$

In particular, $\|\varphi(z)\| \leq 1$ for each $z \in \Omega$.

This result is well known, but we sketch a proof for completeness.

Proof. To prove (12), we use the identity

$$
\left(\begin{array}{ll}
A^{*} A+C^{*} C & A^{*} B+C^{*} D \\
B^{*} A+D^{*} C & B^{*} B+D^{*} D
\end{array}\right)=\left(\begin{array}{ll}
A^{*} & C^{*} \\
B^{*} & D^{*}
\end{array}\right)\left(\begin{array}{ll}
A & B \\
C & D
\end{array}\right)=\left(\begin{array}{ll}
I & 0 \\
0 & I
\end{array}\right) .
$$

Thus

$$
\begin{aligned}
I-\varphi(z)^{*} \varphi(w) & \\
=I- & {\left[D^{*}+B^{*}\left(I-Z(z)^{*} A^{*}\right)^{-1} Z(z)^{*} C^{*}\right]\left[D+C Z(w)(I-A Z(w))^{-1} B\right] } \\
=I- & D^{*} D-D^{*} C Z(w)(I-A Z(w))^{-1} B-B^{*}\left(I-Z(z)^{*} A^{*}\right)^{-1} Z(z)^{*} C^{*} D \\
& -B^{*}\left(I-Z(z)^{*} A^{*}\right)^{-1} Z(z)^{*} C^{*} C Z(w)(I-A Z(w))^{-1} B
\end{aligned}
$$




$$
\begin{aligned}
& =B^{*} B+B^{*} A Z(w)(I-A Z(w))^{-1} B+B^{*}\left(I-Z(z)^{*} A^{*}\right)^{-1} Z(z)^{*} A^{*} B \\
& -B^{*}\left(I-Z(z)^{*} A^{*}\right)^{-1} Z(z)^{*}\left(I-A^{*} A\right) Z(w)(I-A Z(w))^{-1} B \\
& =B^{*}\left(I-Z(z)^{*} A^{*}\right)^{-1}\left\{\left(I-Z(z)^{*} A^{*}\right)(I-A Z(w))+\left(I-Z(z)^{*} A^{*}\right) A Z(w)\right. \\
& \left.+Z(z)^{*} A^{*}(I-A Z(w))-Z(z)^{*}\left(I-A^{*} A\right) Z(w)\right\}(I-A Z(w))^{-1} B \\
& =B^{*}\left(I-Z(z)^{*} A^{*}\right)^{-1}\left(I-Z(z)^{*} Z(w)\right)(I-A Z(w))^{-1} B,
\end{aligned}
$$

proving (12). In a similar way, we have

$$
\varphi(z)=D+C\left(I_{\mathcal{H}}-Z(z) A\right)^{-1} Z(z) B
$$

and

$$
\left(\begin{array}{ll}
A A^{*}+B B^{*} & A C^{*}+B D^{*} \\
C A^{*}+D B^{*} & C C^{*}+D D^{*}
\end{array}\right)=\left(\begin{array}{cc}
A & B \\
C & D
\end{array}\right)\left(\begin{array}{ll}
A^{*} & C^{*} \\
B^{*} & D^{*}
\end{array}\right)=\left(\begin{array}{ll}
I & 0 \\
0 & I
\end{array}\right) .
$$

Parallel calculations using these identities yield (13).

The next lemma uses the differentiation rules

$$
\frac{\partial}{\partial z_{j}}[F G]=\frac{\partial F}{\partial z_{j}} G+F \frac{\partial G}{\partial z_{j}}, \quad \frac{\partial}{\partial z_{j}} F^{-1}=-F^{-1} \frac{\partial F}{\partial z_{j}} F^{-1},
$$

which hold for any operator-valued functions for which the operations are defined.

Lemma 2. Let $\varphi$ have the form (8) where (9) is a unitary operator and $Z$ satisfies (10) and (11). Set

$$
L=A\left(I_{\mathcal{H}}-Z A\right)^{-1}=\left(I_{\mathcal{K}}-A Z\right)^{-1} A .
$$

Then for each $j=1, \ldots, d$,

$$
\frac{\partial \varphi}{\partial z_{j}}=C\left(I_{\mathcal{H}}-Z A\right)^{-1} E_{j}\left(I_{\mathcal{K}}-A Z\right)^{-1} B
$$

For every $n \geq 2$ and any $k_{1}, \ldots, k_{n}$ in $\{1,2, \ldots, d\}$,

$$
\frac{\partial^{n} \varphi}{\partial z_{k_{n}} \cdots \partial z_{k_{1}}}=C\left(I_{\mathcal{H}}-Z A\right)^{-1} \sum_{\sigma} E_{k_{\sigma(1)}} L E_{k_{\sigma(2)}} L \cdots L E_{k_{\sigma(n)}}\left(I_{\mathcal{K}}-A Z\right)^{-1} B
$$

where the summation is over all permutations $\sigma$ of $\{1,2, \ldots, n\}$.

Proof. Note that

$$
\begin{aligned}
& \frac{\partial}{\partial z_{j}}(I-Z A)^{-1}=-(I-Z A)^{-1}\left(-E_{j} A\right)(I-Z A)^{-1}=(I-Z A)^{-1} E_{j} L \\
& \frac{\partial}{\partial z_{j}}(I-A Z)^{-1}=-(I-A Z)^{-1}\left(-A E_{j}\right)(I-A Z)^{-1}=L E_{j}(I-A Z)^{-1},
\end{aligned}
$$

and

$$
\begin{aligned}
\frac{\partial L}{\partial z_{j}}=\frac{\partial}{\partial z_{j}} & A(I-Z A)^{-1} \\
& =-A(I-Z A)^{-1}\left(-E_{j} A\right)(I-Z A)^{-1}=L E_{j} L
\end{aligned}
$$


By (17),

$$
\begin{aligned}
\frac{\partial \varphi}{\partial z_{j}} & =C E_{j}(I-A Z)^{-1} B+C Z L E_{j}(I-A Z)^{-1} B \\
& =C\left[I+Z(I-A Z)^{-1} A\right] E_{j}(I-A Z)^{-1} B \\
& =C(I-Z A)^{-1} E_{j}(I-A Z)^{-1} B
\end{aligned}
$$

which is (14). Thus by (16) and (17),

$$
\begin{aligned}
\frac{\partial^{2} \varphi}{\partial z_{k_{2}} \partial z_{k_{1}}}= & \frac{\partial}{\partial z_{k_{2}}} C(I-Z A)^{-1} E_{k_{1}}(I-A Z)^{-1} B \\
= & C(I-Z A)^{-1} E_{k_{2}} L E_{k_{1}}(I-A Z)^{-1} B \\
& \quad+C(I-Z A)^{-1} E_{k_{1}} L E_{k_{2}}(I-A Z)^{-1} B \\
= & C(I-Z A)^{-1} \sum_{\sigma} E_{k_{\sigma(1)}} L E_{k_{\sigma(2)}}(I-A Z)^{-1} B
\end{aligned}
$$

where the summation is over all permutations $\sigma$ of $\{1,2\}$. This is (15) for $n=2$. Assume that (15) is true for some $n \geq 2$. Writing $\sigma$ for an arbitrary permutation of $\{1,2, \ldots, n\}$ and using (16), (17), and (18), we obtain

$$
\begin{aligned}
\frac{\partial}{\partial z_{k_{n+1}} \partial z_{k_{n}} \cdots \partial z_{k_{1}}} & \frac{\partial}{\partial z_{k_{n+1}}}\left\{C(I-Z A)^{-1} \sum_{\sigma} E_{k_{\sigma(1)}} L E_{k_{\sigma(2)}} L \cdots L E_{k_{\sigma(n)}}(I-A Z)^{-1} B\right\} \\
= & C(I-Z A)^{-1} E_{k_{n+1}} L \sum_{\sigma} E_{k_{\sigma(1)}} L E_{k_{\sigma(2)}} L \cdots L E_{k_{\sigma(n)}}(I-A Z)^{-1} B \\
& +C(I-Z A)^{-1}\left\{\frac{\partial}{\partial z_{k_{n+1}}} \sum_{\sigma} E_{k_{\sigma(1)}} L E_{k_{\sigma(2)}} L \cdots L E_{k_{\sigma(n)}}\right\}(I-A Z)^{-1} B \\
& +C(I-Z A)^{-1} \sum_{\sigma} E_{k_{\sigma(1)}} L E_{k_{\sigma(2)}} L \cdots L E_{k_{\sigma(n)}} L E_{k_{n+1}}(I-A Z)^{-1} B \\
=C(I-Z A)^{-1}\left\{\left[E_{k_{n+1}} L\right] \sum_{\sigma} E_{k_{\sigma(1)}} L E_{k_{\sigma(2)}} L \cdots L E_{k_{\sigma(n)}}\right. & \\
& +\sum_{\sigma} E_{k_{\sigma(1)}}\left[L E_{k_{n+1}} L\right] E_{k_{\sigma(2)}} L \cdots L E_{k_{\sigma(n)}} \\
& +\sum_{\sigma} E_{k_{\sigma(1)}} L E_{k_{\sigma(2)}}\left[L E_{k_{n+1}} L\right] \cdots L E_{k_{\sigma(n)}} \\
& +\cdots+\sum_{\sigma} E_{k_{\sigma(1)}} L E_{k_{\sigma(2)}} L \cdots\left[L E_{k_{n+1}} L\right] E_{k_{\sigma(n)}} \\
& \left.+\sum_{\sigma} E_{k_{\sigma(1)}} L E_{k_{\sigma(2)}} L \cdots L E_{k_{\sigma(n)}}\left[L E_{k_{n+1}}\right]\right\}(I-A Z)^{-1} B .
\end{aligned}
$$


This has the form (15) with $n$ replaced by $n+1$. By induction, (15) holds for all $n \geq 2$.

The norm estimates in Lemmas 3 and 4 are understood to be in the pointwise sense.

Lemma 3. Let $\varphi$ have the form (8) where (9) is a unitary operator and $Z$ satisfies (10) and (11). For each $j=1, \ldots, d$,

$$
\begin{aligned}
& \left\|E_{j}\left(I_{\mathcal{K}}-A Z\right)^{-1} B\right\| \leq\left\|I_{\mathcal{F}}-\varphi^{*} \varphi\right\|^{\frac{1}{2}}\left\|E_{j}\left(I_{\mathcal{K}}-Z^{*} Z\right)^{-1} E_{j}^{*}\right\|^{\frac{1}{2}} \\
& \left\|C\left(I_{\mathcal{H}}-Z A\right)^{-1} E_{j}\right\| \leq\left\|I_{\mathcal{G}}-\varphi \varphi^{*}\right\|^{\frac{1}{2}}\left\|E_{j}^{*}\left(I_{\mathcal{H}}-Z Z^{*}\right)^{-1} E_{j}\right\|^{\frac{1}{2}}
\end{aligned}
$$

Moreover,

$$
\begin{aligned}
& \left\|\left(I_{\mathcal{K}}-A Z\right)^{-1} B\right\| \leq \sqrt{\frac{\left\|I_{\mathcal{F}}-\varphi^{*} \varphi\right\|}{1-\|Z\|^{2}}}, \\
& \left\|C\left(I_{\mathcal{H}}-Z A\right)^{-1}\right\| \leq \sqrt{\frac{\left\|I_{\mathcal{G}}-\varphi \varphi^{*}\right\|}{1-\|Z\|^{2}}} .
\end{aligned}
$$

Proof. By (12), $\left\|\left(I-\varphi^{*} \varphi\right)^{\frac{1}{2}}\right\|=\left\|\left(I-Z^{*} Z\right)^{\frac{1}{2}}(I-A Z)^{-1} B\right\|$. Therefore

$$
\begin{aligned}
\left\|E_{j}(I-A Z)^{-1} B\right\| & =\left\|E_{j}\left(I-Z^{*} Z\right)^{-\frac{1}{2}}\left(I-Z^{*} Z\right)^{\frac{1}{2}}(I-A Z)^{-1} B\right\| \\
& \leq\left\|E_{j}\left(I-Z^{*} Z\right)^{-\frac{1}{2}}\right\|\left\|I-\varphi^{*} \varphi\right\|^{\frac{1}{2}} \\
& =\left\|I-\varphi^{*} \varphi\right\|^{\frac{1}{2}}\left\|E_{j}\left(I-Z^{*} Z\right)^{-1} E_{j}^{*}\right\|^{\frac{1}{2}},
\end{aligned}
$$

which is (19). Similarly, using (13) we obtain

$$
\begin{aligned}
\left\|C(I-Z A)^{-1} E_{j}\right\| & =\left\|E_{j}^{*}\left(I-A^{*} Z^{*}\right)^{-1} C^{*}\right\| \\
& =\left\|E_{j}^{*}\left(I-Z Z^{*}\right)^{-\frac{1}{2}}\left(I-Z Z^{*}\right)^{\frac{1}{2}}\left(I-A^{*} Z^{*}\right)^{-1} C^{*}\right\| \\
& \leq\left\|E_{j}^{*}\left(I-Z Z^{*}\right)^{-\frac{1}{2}}\right\|\left\|\left(I-\varphi \varphi^{*}\right)^{\frac{1}{2}}\right\| \\
& =\left\|I-\varphi \varphi^{*}\right\|^{\frac{1}{2}}\left\|E_{j}^{*}\left(I-Z Z^{*}\right)^{-1} E_{j}\right\|^{\frac{1}{2}},
\end{aligned}
$$

which is (201). To prove (21) and (22) we proceed in the same way without the factors $E_{j}$ and $E_{j}^{*}$, and at the last stage we use the inequality

$$
\left\|\left(I-Z^{*} Z\right)^{-\frac{1}{2}}\right\|^{2}=\left\|\left(I-Z^{*} Z\right)^{-1}\right\| \leq \sum_{k=0}^{\infty}\left\|Z^{*} Z\right\|^{k}=\frac{1}{1-\|Z\|^{2}}
$$

and its companion $\left\|\left(I-Z Z^{*}\right)^{-\frac{1}{2}}\right\|^{2} \leq 1 /\left(1-\|Z\|^{2}\right)$. 
Lemma 4. Let $\varphi$ have the form (8) where (9) is a unitary operator and $Z$ satisfies (10) and (11).

(i) For each $j=1, \ldots, d$,

$$
\begin{aligned}
\left\|\frac{\partial \varphi}{\partial z_{j}}\right\| \leq \frac{\left\|I_{\mathcal{F}}-\varphi^{*} \varphi\right\|^{\frac{1}{2}}\left\|I_{\mathcal{G}}-\varphi \varphi^{*}\right\|^{\frac{1}{2}}}{\left(1-\|Z\|^{2}\right)^{\frac{1}{2}}} . \\
\cdot \min \left\{\left\|E_{j}\left(I_{\mathcal{K}}-Z^{*} Z\right)^{-1} E_{j}^{*}\right\|^{\frac{1}{2}},\left\|E_{j}^{*}\left(I_{\mathcal{H}}-Z Z^{*}\right)^{-1} E_{j}\right\|^{\frac{1}{2}}\right\} .
\end{aligned}
$$

(ii) For every $n \geq 2$ and any $k_{1}, \ldots, k_{n}$ in $\{1,2, \ldots, d\}$,

$$
\begin{gathered}
\left\|\frac{\partial^{n} \varphi}{\partial z_{k_{n}} \cdots \partial z_{k_{1}}}\right\| \leq(n-2) ! \frac{\left\|I_{\mathcal{F}}-\varphi^{*} \varphi\right\|^{\frac{1}{2}}\left\|I_{\mathcal{G}}-\varphi \varphi^{*}\right\|^{\frac{1}{2}}}{(1-\|Z\|)^{n-1}} . \\
\cdot \sum_{\substack{p, q=1 \\
p \neq q}}^{n}\left\|E_{k_{p}}\left(I_{\mathcal{K}}-Z^{*} Z\right)^{-1} E_{k_{p}}^{*}\right\|^{\frac{1}{2}}\left\|E_{k_{q}}^{*}\left(I_{\mathcal{H}}-Z Z^{*}\right)^{-1} E_{k_{q}}\right\|^{\frac{1}{2}} .
\end{gathered}
$$

Proof. (i) By (14), (19) , and (22),

$$
\begin{aligned}
\left\|\frac{\partial \varphi}{\partial z_{j}}\right\| & \leq\left\|C(I-Z A)^{-1}\right\|\left\|E_{j}(I-A Z)^{-1} B\right\| \\
& \leq \frac{\left\|I-\varphi^{*} \varphi\right\|^{\frac{1}{2}}\left\|I-\varphi \varphi^{*}\right\|^{\frac{1}{2}}}{\left(1-\|Z\|^{2}\right)^{\frac{1}{2}}}\left\|E_{j}\left(I-Z^{*} Z\right)^{-1} E_{j}^{*}\right\|^{\frac{1}{2}} .
\end{aligned}
$$

Using (20) and (21), we get

$$
\begin{aligned}
\left\|\frac{\partial \varphi}{\partial z_{j}}\right\| & \leq\left\|C(I-Z A)^{-1} E_{j}\right\|\left\|(I-A Z)^{-1} B\right\| \\
& \leq \frac{\left\|I-\varphi^{*} \varphi\right\|^{\frac{1}{2}}\left\|I-\varphi \varphi^{*}\right\|^{\frac{1}{2}}}{\left(1-\|Z\|^{2}\right)^{\frac{1}{2}}}\left\|E_{j}^{*}\left(I-Z Z^{*}\right)^{-1} E_{j}\right\|^{\frac{1}{2}} .
\end{aligned}
$$

The inequality in (i) follows.

(ii) By (15), (19), and (20),

$$
\begin{aligned}
& \left\|\frac{\partial^{n} \varphi}{\partial z_{k_{n}} \cdots \partial z_{k_{1}}}\right\| \\
& \quad=\left\|\sum_{\sigma} C(I-Z A)^{-1} E_{k_{\sigma(1)}} L E_{k_{\sigma(2)}} L \cdots E_{k_{\sigma(n-1)}} L E_{k_{\sigma(n)}}(I-A Z)^{-1} B\right\| \\
& \leq \sum_{\sigma}\left\|C(I-Z A)^{-1} E_{k_{\sigma(1)}}\right\|\|L\|^{n-1}\left\|E_{k_{\sigma(n)}}(I-A Z)^{-1} B\right\| \\
& \leq\|L\|^{n-1} \sum_{\sigma}\left\|I-\varphi \varphi^{*}\right\|^{\frac{1}{2}}\left\|E_{k_{\sigma(1)}^{*}}\left(I-Z Z^{*}\right)^{-1} E_{k_{\sigma(1)}}\right\|^{\frac{1}{2}} \\
& \cdot\left\|I-\varphi^{*} \varphi\right\|^{\frac{1}{2}}\left\|E_{k_{\sigma(n)}}\left(I-Z^{*} Z\right)^{-1} E_{k_{\sigma(n)}^{*}}\right\|^{\frac{1}{2}} .
\end{aligned}
$$


Here the summations run over all permutations $\sigma$ of $\{1, \ldots, n\}$. Since

$$
\|L\|=\left\|\sum_{k=0}^{\infty} A[Z A]^{k}\right\| \leq \sum_{k=0}^{\infty}\|Z\|^{k}=\frac{1}{1-\|Z\|}
$$

we obtain

$$
\begin{aligned}
\left\|\frac{\partial^{n} \varphi}{\partial z_{k_{n}} \cdots \partial z_{k_{1}}}\right\| \leq & \frac{\left\|I-\varphi^{*} \varphi\right\|^{\frac{1}{2}}\left\|I-\varphi \varphi^{*}\right\|^{\frac{1}{2}}}{(1-\|Z\|)^{n-1}} \cdot \\
& \cdot \sum_{\sigma}\left\|E_{k_{\sigma(1)}^{*}}^{*}\left(I-Z Z^{*}\right)^{-1} E_{k_{\sigma(1)}}\right\|^{\frac{1}{2}}\left\|E_{k_{\sigma(n)}}\left(I-Z^{*} Z\right)^{-1} E_{k_{\sigma(n)}}^{*}\right\|^{\frac{1}{2}} \\
= & (n-2) ! \frac{\left\|I-\varphi^{*} \varphi\right\|^{\frac{1}{2}}\left\|I-\varphi \varphi^{*}\right\|^{\frac{1}{2}}}{(1-\|Z\|)^{n-1}} \cdot \\
& \cdot \sum_{\substack{p, q=1 \\
p \neq q}}^{n}\left\|E_{k_{p}}\left(I-Z^{*} Z\right)^{-1} E_{k_{p}}^{*}\right\|^{\frac{1}{2}}\left\|E_{k_{q}}^{*}\left(I-Z Z^{*}\right)^{-1} E_{k_{q}}\right\|^{\frac{1}{2}} .
\end{aligned}
$$

This proves the inequality in (ii).

\section{The Schur-Agler class on the polydisk}

To begin, we apply the esimates derived in $\$ 2$ to complex-valued functions on the polydisk $\mathbb{D}^{d}$. Set

$$
\|z\|_{\infty}=\max _{j=1, \ldots, d}\left|z_{j}\right|, \quad z=\left(z_{1}, \ldots, z_{d}\right) \in \mathbb{C}^{d} .
$$

Theorem 5. Let $\varphi(z)$ be a complex-valued function in the Schur-Agler class on the polydisk $\mathbb{D}^{d}$. For each $z \in \mathbb{D}^{d}$ and $j=1, \ldots, d$,

$$
\left|\frac{\partial \varphi}{\partial z_{j}}\right| \leq \frac{1-|\varphi(z)|^{2}}{\sqrt{1-\left|z_{j}\right|^{2}} \sqrt{1-\|z\|_{\infty}^{2}}} .
$$

For each $z \in \mathbb{D}^{d}$, every $n \geq 2$, and any $k_{1}, \ldots, k_{n}$ in $\{1,2, \ldots, d\}$,

$$
\left|\frac{\partial^{n} \varphi}{\partial z_{k_{n}} \cdots \partial z_{k_{1}}}\right| \leq(n-2) ! \frac{1-|\varphi(z)|^{2}}{\left(1-\|z\|_{\infty}\right)^{n-1}} \sum_{\substack{p, q=1 \\ p \neq q}}^{n} \frac{1}{\sqrt{1-\left|z_{k_{p}}\right|^{2}} \sqrt{1-\left|z_{k_{q}}\right|^{2}}} .
$$

Proof. Represent $\varphi(z)$ in the form (8) with $\mathcal{F}=\mathcal{G}=\mathbb{C}$, the complex numbers in the Euclidean metric, and choose $\Omega=\mathbb{D}^{d}$. Following $\$ 1.1$, we assume that the Hilbert spaces $\mathcal{H}$ and $\mathcal{K}$ satisfy $\mathcal{K}=\mathcal{H}=\mathcal{H}_{1} \oplus \cdots \oplus \mathcal{H}_{d}$, and that $Z(z)=$ $z_{1} E_{1}+\cdots+z_{d} E_{d}$, where $E_{j}$ is the orthogonal projection onto $\mathcal{H}_{j}, j=1, \ldots, d$. Then $\|Z(z)\| \leq\|z\|_{\infty}$. For any $j=1, \ldots, d$,

$$
E_{j}\left(I-Z(z) Z(z)^{*}\right)^{-1} E_{j}^{*}=E_{j}^{*}\left(I-Z(z)^{*} Z(z)\right)^{-1} E_{j}=\frac{1}{1-\left|z_{j}\right|^{2}} E_{j},
$$

and therefore

$$
\left\|E_{j}\left(I-Z(z) Z(z)^{*}\right)^{-1} E_{j}^{*}\right\|^{\frac{1}{2}}=\left\|E_{j}^{*}\left(I-Z(z)^{*} Z(z)\right)^{-1} E_{j}\right\|^{\frac{1}{2}} \leq \frac{1}{\sqrt{1-\left|z_{j}\right|^{2}}} .
$$


Now apply Lemma 4 .

Recall that the Schur-Agler class on $\mathbb{D}^{2}$ is the full Schur class of analytic functions which are defined and bounded by one on $\mathbb{D}^{2}$.

Corollary 6. Let $\varphi(z)$ be any complex-valued function which is analytic and bounded by one on $\mathbb{D}^{2}$. For each $z \in \mathbb{D}^{2}$ and $j=1,2$,

$$
\left|\frac{\partial \varphi}{\partial z_{j}}\right| \leq \frac{1-|\varphi(z)|^{2}}{\sqrt{1-\left|z_{j}\right|^{2}} \sqrt{1-\|z\|_{\infty}^{2}}} .
$$

For each $z \in \mathbb{D}^{2}$ and any nonnegative integers $n_{1}, n_{2}$ with $n=n_{1}+n_{2} \geq 2$,

$$
\begin{aligned}
&\left|\frac{\partial^{n} \varphi}{\partial z_{2}^{n_{2}} \partial z_{1}^{n_{1}}}\right| \leq(n-2) ! \frac{1-|\varphi(z)|^{2}}{\left(1-\|z\| \|_{\infty}\right)^{n-1}} . \\
& \cdot\left[\frac{n_{1}^{2}-n_{1}}{1-\left|z_{1}\right|^{2}}+\frac{2 n_{1} n_{2}}{\sqrt{1-\left|z_{1}\right|^{2}} \sqrt{1-\left|z_{2}\right|^{2}}}+\frac{n_{2}^{2}-n_{2}}{1-\left|z_{2}\right|^{2}}\right] .
\end{aligned}
$$

Proof. The inequality (26) is a repeat of (24). To deduce (27) from (25), write

$$
\left(k_{1}, \ldots, k_{n}\right)=(\overbrace{1, \ldots, 1}^{n_{1}}, \overbrace{2, \ldots, 2}^{n_{2}}),
$$

and collect similar terms.

The next result takes advantage of the special form of the polydisk.

Theorem 7. Let $\varphi(z)$ be a complex-valued function in the Schur-Agler class on the polydisk $\mathbb{D}^{d}$. Then for each $z \in \mathbb{D}^{d}$ and any nonnegative integers $n_{1}, \ldots, n_{d}$ which are not all zero,

$$
\left|\frac{\partial^{n} \varphi}{\partial z_{d}^{n_{d}} \cdots \partial z_{1}^{n_{1}}}\right| \leq n_{1} ! \cdots n_{d} ! \frac{1-|\varphi(z)|^{2}}{\left(1-\|z\|_{\infty}^{2}\right)\left(1-\|z\|_{\infty}\right)^{n-1}},
$$

where $n=n_{1}+\cdots+n_{d}$.

The weaker inequality

$$
\left|\frac{\partial^{n} \varphi}{\partial z_{d}^{n_{d}} \cdots \partial z_{1}^{n_{1}}}\right| \leq n ! \frac{1-|\varphi(z)|^{2}}{\left(1-\|z\|_{\infty}^{2}\right)\left(1-\|z\|_{\infty}\right)^{n-1}},
$$

follows from Theorem 5. In fact, when $n=1$ this is immediate from (24). Suppose $n \geq 2$. Set

$$
\left(k_{1}, \ldots, k_{n}\right)=(\overbrace{1, \ldots, 1}^{n_{1}}, \overbrace{2, \ldots, 2}^{n_{2}}, \ldots, \overbrace{d, \ldots, d}^{n_{d}}) .
$$

By (25),

$$
\begin{aligned}
& \left|\frac{\partial^{n} \varphi}{\partial z_{d}^{n_{d}} \cdots \partial z_{1}^{n_{1}}}\right|=\left|\frac{\partial^{n} \varphi}{\partial z_{k_{n}} \cdots \partial z_{k_{1}}}\right| \leq(n-2) ! \frac{1-|\varphi(z)|^{2}}{\left(1-\|z\|_{\infty}\right)^{n-1}} \sum_{\substack{p, q=1 \\
p \neq q}}^{n} \frac{1}{1-\|z\|_{\infty}^{2}} \\
= & (n-2) ! \frac{1-|\varphi(z)|^{2}}{\left(1-\|z\|_{\infty}^{2}\right)\left(1-\|z\|_{\infty}\right)^{n-1}}\left(n^{2}-n\right)=n ! \frac{1-|\varphi(z)|^{2}}{\left(1-\|z\|_{\infty}^{2}\right)\left(1-\|z\|_{\infty}\right)^{n-1}},
\end{aligned}
$$


yielding (29).

A variation on the method, which is valid for the polydisk case but not generally, is needed to prove (28).

Lemma 8. Let $L \in \mathcal{L}(\mathcal{H})$, where $\mathcal{H}$ is a Hilbert space. Assume that $\mathcal{H}=\mathcal{H}_{1} \oplus$ $\cdots \oplus \mathcal{H}_{d}$, and let $E_{1}, \ldots, E_{d}$ be the orthogonal projections onto the summands. Suppose that $n=n_{1}+\cdots+n_{d}$ for some nonnegative integers $n_{1}, \ldots, n_{d}$. Assume that $n \geq 2$, and set

$$
K=\sum E_{j_{1}} L E_{j_{2}} L \cdots E_{j_{n-1}} L E_{j_{n}},
$$

where the summation is over all distinct arrangements $\left(j_{1}, j_{2}, \ldots, j_{n}\right)$ of the tuple

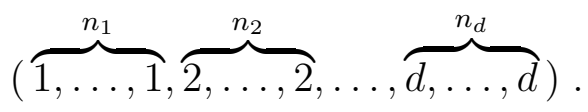

Then

$$
\|K\| \leq\|L\|^{n-1} .
$$

The number of terms in (31) is the multinomial coefficient

$$
\left(n ; n_{1}, n_{2}, \ldots, n_{d}\right)=\frac{n !}{n_{1} ! n_{2} ! \cdots n_{d} !} .
$$

Lemma 8 is not stated in maximum generality, but it will suit our application.

Proof of Lemma 8. We first illustrate the method for the example $n_{1}=3$, $n_{2}=2$, and $d=2$. Write

$$
K=K[1,1,1,2,2]=\sum E_{j_{1}} L E_{j_{2}} L E_{j_{3}} L E_{j_{4}} L E_{j_{5}},
$$

where the summation is over all distinct arrangements of $(1,1,1,2,2)$ :

$(1,1,1,2,2),(1,1,2,1,2),(1,1,2,2,1),(1,2,1,1,2),(1,2,1,2,1),(1,2,2,1,1)$,

$(2,1,1,1,2),(2,1,1,2,1),(2,1,2,1,1),(2,2,1,1,1)$.

Define $K[1,1,2,2], K[1,2,2], \ldots$ in a similar way. For example, $K[1,1,2,2]$ is the sum of all terms $E_{j_{1}} L E_{j_{2}} L E_{j_{3}} L E_{j_{4}}$ over the set of distinct arrangements of $(1,1,2,2)$. Now split the sum (34) into two parts, the first part consisting of all terms with $j_{1}=1$ and the second consisting of all terms with $j_{1}=2$. The result is

$$
K=E_{1} L K[1,1,2,2]+E_{2} L K[1,1,1,2] .
$$

Since $E_{1}$ and $E_{2}$ have orthogonal ranges, for any $f \in \mathcal{H}$,

$$
\begin{aligned}
\|K f\|^{2} & =\left\|E_{1} L K[1,1,2,2] f\right\|^{2}+\left\|E_{2} L K[1,1,1,2] f\right\|^{2} \\
& \leq\|L\|^{2}\left\{\|K[1,1,2,2] f\|^{2}+\|K[1,1,1,2] f\|^{2}\right\} .
\end{aligned}
$$

Next decompose $K[1,1,2,2] f$ and $K[1,1,1,2] f$ in the same way:

$$
\begin{aligned}
&\|K f\|^{2} \leq\|L\|^{2}\left\{\left\|E_{1} L K[1,2,2] f\right\|^{2}+\left\|E_{2} L K[1,1,2] f\right\|^{2}\right. \\
&\left.+\left\|E_{1} L K[1,1,2] f\right\|^{2}+\left\|E_{2} L K[1,1,1] f\right\|^{2}\right\} .
\end{aligned}
$$


The two middle terms in the last expression combine and give

$$
\begin{aligned}
\left\|E_{2} L K[1,1,2] f\right\|^{2}+\left\|E_{1} L K[1,1,2] f\right\|^{2} & =\left\|\left(E_{2}+E_{1}\right) L K[1,1,2] f\right\|^{2} \\
& \leq\|L\|^{2}\|K[1,1,2] f\|^{2},
\end{aligned}
$$

because $E_{1}+E_{2}$ is also an orthogonal projection. Thus

$$
\|K f\|^{2} \leq\|L\|^{4}\left\{\|K[1,2,2] f\|^{2}+\|K[1,1,2] f\|^{2}+\|K[1,1,1] f\|^{2}\right\} .
$$

Continuing in this way, we obtain

$$
\begin{aligned}
\|K f\|^{2} \leq & \|L\|^{4}\left\{\left\|E_{1} L K[2,2] f\right\|^{2}+\left\|E_{2} L K[1,2] f\right\|^{2}\right. \\
& \left.\quad+\left\|E_{1} L K[1,2] f\right\|^{2}+\left\|E_{2} L K[1,1] f\right\|^{2}+\left\|E_{1} L K[1,1] f\right\|^{2}\right\} \\
\leq & \|L\|^{6}\left\{\|K[2,2] f\|^{2}+\|K[1,2] f\|^{2}+\|K[1,1] f\|^{2}\right\} \\
= & \|L\|^{6}\left\{\left\|E_{2} L E_{2} f\right\|^{2}+\left(\left\|E_{1} L E_{2} f\right\|^{2}+\left\|E_{2} L E_{1} f\right\|^{2}\right)+\left\|E_{1} L E_{1} f\right\|^{2}\right\} \\
\leq & \|L\|^{8}\left\{\left\|E_{2} f\right\|^{2}+\left\|E_{1} f\right\|^{2}\right\} \\
= & \|L\|^{8}\|f\|^{2} .
\end{aligned}
$$

Thus $\|K\| \leq\|L\|^{4}$, which proves the lemma for the example.

In the general case, we consider any $f \in \mathcal{H}$ and write

$$
\begin{aligned}
K f=E_{1} L K[\overbrace{1, \ldots, 1}^{n_{1}-1} & , \overbrace{2, \ldots, 2}^{n_{2}}, \ldots, \overbrace{d, \ldots, d}^{n_{d}}] f \\
& +E_{2} L K[\overbrace{1, \ldots, 1}^{n_{1}} \overbrace{2, \ldots, 2}^{n_{2}-1}, \ldots, \overbrace{d, \ldots, d}^{n_{d}}] f \\
& +\cdots+E_{d} L K[\overbrace{1, \ldots, 1}^{n_{1}}, \overbrace{2, \ldots, 2}^{n_{2}}, \ldots, \overbrace{d, \ldots, d}^{n_{d}-1}] f .
\end{aligned}
$$

As in the example, we use orthogonality and repeatedly break down all expressions into smaller and smaller patterns which have the same length at each stage. Whenever a pattern $K\left[p_{1}, \ldots, p_{\ell}\right]$ appears more than once, it is in a group

$$
E_{q_{1}} L K\left[p_{1}, \ldots, p_{\ell}\right] f, E_{q_{2}} L K\left[p_{1}, \ldots, p_{\ell}\right] f, \ldots, E_{q_{m}} L K\left[p_{1}, \ldots, p_{\ell}\right] f
$$

with distinct $q_{1}, q_{2}, \ldots, q_{m}$ in $\{1, \ldots, d\}$. Since these expressions are orthogonal, the group can be collapsed. In the end, we obtain $\|K f\|^{2} \leq\|L\|^{2(n-1)}\|f\|^{2}$. By the arbitrariness of $f,(33)$ follows.

Proof of Theorem 7. We use the representation (8) of $\varphi(z)$ with the same choices of Hilbert spaces and operators as in the proof of Theorem 5. When $n=1$, (28) is immediate from (14), (21), and (22). Suppose $n \geq 2$. By (15) 
applied with $\left(k_{1}, k_{2}, \ldots, k_{n}\right)$ as in (30),

$$
\begin{aligned}
\frac{\partial^{n} \varphi}{\partial z_{d}^{n_{d}} \cdots \partial z_{1}^{n_{1}}} & \\
= & \frac{\partial^{n} \varphi}{\partial z_{k_{n}} \cdots \partial z_{k_{1}}} \\
= & C\left(I_{\mathcal{H}}-Z(z) A\right)^{-1} \cdot \sum_{\sigma} E_{k_{\sigma(1)}} L(z) E_{k_{\sigma(2)}} L(z) \cdots L(z) E_{k_{\sigma(n)}} \cdot \\
& \cdot\left(I_{\mathcal{K}}-A Z(z)\right)^{-1} B \\
= & n_{1} ! n_{2} ! \cdots n_{d} ! C\left(I_{\mathcal{H}}-Z(z) A\right)^{-1} \cdot \sum E_{j_{1}} L(z) E_{j_{2}} L(z) \cdots L(z) E_{j_{n}} \\
& \cdot\left(I_{\mathcal{K}}-A Z(z)\right)^{-1} B,
\end{aligned}
$$

where the last summation is over all distinct arrangements $\left(j_{1}, j_{2}, \ldots, j_{n}\right)$ of the tuple (32). The factorials $n_{1} !, n_{2} !, \ldots, n_{d}$ ! account for the permutations of the groups in (30). By Lemma 8 applied with $L=L(z)$,

$$
\left|\frac{\partial^{n} \varphi}{\partial z_{d}^{n_{d}} \cdots \partial z_{1}^{n_{1}}}\right| \leq\left\|C\left(I_{\mathcal{H}}-Z(z) A\right)^{-1}\right\|\|L(z)\|^{n-1}\left\|\left(I_{\mathcal{K}}-A Z(z)\right)^{-1} B\right\| .
$$

Then (28) follows from (21), (22), and (23).

A theorem of $\mathrm{F}$. Wiener [13, 14, 19] asserts that if $\varphi(z)$ is a complex-valued function which is analytic and bounded by one on $\mathbb{D}$, and if $\varphi(z)=\sum_{k=0}^{\infty} c_{k} z^{k}$, then $\left|c_{k}\right| \leq 1-\left|c_{0}\right|^{2}$ for every $k \geq 1$. An immediate consequence of Theorem 7 is an analog for the Schur-Agler class on the polydisk.

Corollary 9. Let $\varphi(z)$ be a complex-valued function in the Schur-Agler class on the polydisk $\mathbb{D}^{d}$. If

$$
\varphi(z)=\sum_{k_{1}, \ldots, k_{d}=0}^{\infty} c_{k_{1} \cdots k_{d}} z_{1}^{k_{1}} \cdots z_{d}^{k_{d}}
$$

then $\left|c_{n_{1} \cdots n_{d}}\right| \leq 1-\left|c_{0 \cdots 0}\right|^{2}$ for all nonnegative integers $n_{1}, \ldots, n_{d}$ which are not all zero.

The question arises if one of the estimates (25) or (28) is always better than the other. This is not the case. Take $d=2, n \geq 2$, and use the equivalent form (27) for (25). For $z=(0,0)$, (28) is a better estimate than (27) except in trivial cases. However, it is not hard to see that when $\left|z_{2}\right|>\left|z_{1}\right|$ and $n_{2}=0$, the reverse is true.

\section{The Schur-Agler class on the unit ball}

In a similar way we can specialize the results of \$2 to the Schur-Agler class on the unit ball $\mathbb{B}_{d}$. For any $z=\left(z_{1}, \ldots, z_{d}\right)$ in $\mathbb{C}^{d}$, write

$$
\|z\|_{2}=\langle z, z\rangle^{\frac{1}{2}}=\sqrt{\left|z_{1}\right|^{2}+\cdots+\left|z_{d}\right|^{2}}
$$


and

$$
\hat{z}_{j}=\left(z_{1}, \ldots, z_{j-1}, 0, z_{j+1}, \ldots, z_{d}\right) .
$$

Theorem 10. Let $\varphi(z)$ be a complex-valued function in the Schur-Agler class on the unit ball. For each $z \in \mathbb{B}_{d}$ and any nonnegative integers $n_{1}, \ldots, n_{d}$ which are not all zero,

$$
\left|\frac{\partial^{n} \varphi}{\partial z_{d}^{n_{d}} \cdots \partial z_{1}^{n_{1}}}\right| \leq(n-1) ! \frac{1-|\varphi(z)|^{2}}{\left(1-\|z\|_{2}^{2}\right)\left(1-\|z\|_{2}\right)^{n-1}} \sum_{j=1}^{d} n_{j} \sqrt{1-\left\|\hat{z}_{j}\right\|_{2}^{2}},
$$

where $n=n_{1}+\cdots+n_{d}$.

Proof. Represent $\varphi(z)$ in the form (8) with $\mathcal{F}=\mathcal{G}=\mathbb{C}$, and let $\Omega=\mathbb{B}_{d}$. As in $\S 1.2$, we take $\mathcal{K}=\mathcal{H} \oplus \cdots \oplus \mathcal{H}$ with $d$ summands, and we write the elements of $\mathcal{K}$ in column form. Then

$$
Z(z)=\left[\begin{array}{lll}
z_{1} I_{\mathcal{H}} & \cdots & z_{d} I_{\mathcal{H}}
\end{array}\right] \quad \text { and } \quad E_{j}=\left[\begin{array}{lllllll}
0 & \cdots & 0 & I_{\mathcal{H}} & 0 & \cdots & 0
\end{array}\right],
$$

where the identity operator appears in the $j$-th position in the formula for $E_{j}$, $j=1, \ldots, d$. Thus $Z(z) Z(z)^{*}=\|z\|_{2}^{2} I_{\mathcal{H}}$, and hence $\|Z(z)\|=\left\|Z(z) Z(z)^{*}\right\|^{\frac{1}{2}}=$ $\|z\|_{2}$ and

$$
\left\|E_{j}^{*}\left(I-Z(z) Z(z)^{*}\right)^{-1} E_{j}\right\|=\frac{1}{1-\|z\|_{2}^{2}} .
$$

Using $E_{j} Z(z)^{*}=\bar{z}_{j} I_{\mathcal{H}}$ and $E_{j} E_{j}^{*}=I_{\mathcal{H}}$, we get

$$
\begin{aligned}
E_{j}\left(I_{\mathcal{K}}-Z(z)^{*} Z(z)\right)^{-1} E_{j}^{*} \\
\quad=E_{j}\left(I_{\mathcal{K}}+Z(z)^{*} Z(z)+Z(z)^{*} Z(z) Z(z)^{*} Z(z)+\cdots\right) E_{j}^{*} \\
\quad=I_{\mathcal{H}}+\bar{z}_{j} I_{\mathcal{H}} z_{j}+\bar{z}_{j} Z(z) Z(z)^{*} z_{j}+\bar{z}_{j} Z(z) Z(z)^{*} Z(z) Z(z)^{*} z_{j}+\cdots \\
=I_{\mathcal{H}}+\left|z_{j}\right|^{2}\left(I_{\mathcal{H}}-Z(z) Z(z)^{*}\right)^{-1} \\
\quad=\left(1+\frac{\left|z_{j}\right|^{2}}{1-\|z\|_{2}^{2}}\right) I_{\mathcal{H}} .
\end{aligned}
$$

Therefore

$$
\left\|E_{j}\left(I_{\mathcal{K}}-Z(z)^{*} Z(z)\right)^{-1} E_{j}^{*}\right\|=1+\frac{\left|z_{j}\right|^{2}}{1-\|z\|_{2}^{2}}=\frac{1-\left\|\hat{z}_{j}\right\|_{2}^{2}}{1-\|z\|_{2}^{2}} .
$$

By Lemma 4)(i),

$$
\left|\frac{\partial \varphi}{\partial z_{j}}\right| \leq \frac{1-|\varphi(z)|^{2}}{\sqrt{1-\|z\|_{2}^{2}}} \sqrt{\frac{1-\left\|\hat{z}_{j}\right\|_{2}^{2}}{1-\|z\|_{2}^{2}}}=\frac{1-|\varphi(z)|^{2}}{1-\|z\|_{2}^{2}} \sqrt{1-\left\|\hat{z}_{j}\right\|_{2}^{2}},
$$

which is (36) when $n=1$. 
Consider $n \geq 2$ and any $k_{1}, \ldots, k_{n}$ in $\{1,2, \ldots, d\}$. By Lemma 4(ii), (37), and (38),

$$
\begin{aligned}
\left|\frac{\partial^{n} \varphi}{\partial z_{k_{n}} \cdots \partial z_{k_{1}}}\right| & \leq(n-2) ! \frac{1-|\varphi(z)|^{2}}{\left(1-\|z\|_{2}\right)^{n-1}} \sum_{\substack{p, q=1 \\
p \neq q}}^{n} \frac{1}{\sqrt{1-\|z\|_{2}^{2}}} \sqrt{\frac{1-\left\|\hat{z}_{k_{q}}\right\|_{2}^{2}}{1-\|z\|_{2}^{2}}} \\
& =(n-2) ! \frac{1-|\varphi(z)|^{2}}{\left(1-\|z\|_{2}^{2}\right)\left(1-\|z\|_{2}\right)^{n-1}} \sum_{\substack{p, q=1 \\
p \neq q}}^{n} \sqrt{1-\left\|\hat{z}_{k_{q}}\right\|_{2}^{2}} \\
& =(n-1) ! \frac{1-|\varphi(z)|^{2}}{\left(1-\|z\|_{2}^{2}\right)\left(1-\|z\|_{2}\right)^{n-1}} \sum_{j=1}^{n} \sqrt{1-\left\|\hat{z}_{k_{j}}\right\|_{2}^{2}}
\end{aligned}
$$

The inequality (36) follows on choosing $k_{1}, \ldots, k_{n}$ as in (30).

Lemma 8 does not apply directly to the unit ball, but it has a counterpart which is applicable to the unit ball.

Lemma 11. Let $L \in \mathcal{L}(\mathcal{H}, \mathcal{K})$, where $\mathcal{H}$ and $\mathcal{K}$ are Hilbert spaces and $\mathcal{K}=$ $\mathcal{H} \oplus \cdots \oplus \mathcal{H}$ with $d$ summands. For each $j=1, \ldots, d$, let $E_{j} \in \mathcal{L}(\mathcal{K}, \mathcal{H})$ be the operator $E_{j}\left(h_{1} \oplus \cdots \oplus h_{d}\right)=h_{j}$. Suppose that $n=n_{1}+\cdots+n_{d}$ for some nonnegative integers $n_{1}, \ldots, n_{d}$. Assume that $n \geq 2$, and set

$$
K=\sum E_{j_{1}} L E_{j_{2}} L \cdots E_{j_{n-1}} L E_{j_{n}}
$$

where the summation is over all distinct arrangements $\left(j_{1}, j_{2}, \ldots, j_{n}\right)$ of the tuple

$$
(\overbrace{1, \ldots, 1}^{n_{1}}, \overbrace{2, \ldots, 2}^{n_{2}}, \ldots, \overbrace{d, \ldots, d}^{n_{d}})
$$

Then

$$
\|K\| \leq d^{(n-1) / 2}\|L\|^{n-1}
$$

Proof of Lemma 11. The argument is similar to the proof of Lemma 8 . We show how it works when $n_{1}=3, n_{2}=2$, and $d=2$. Write the adjoint of $K$ as

$$
\begin{aligned}
K^{*}=\widetilde{K}[1,1,1,2,2]=\sum E_{j_{5}}^{*} L^{*} E_{j_{4}}^{*} L^{*} E_{j_{3}}^{*} L^{*} E_{j_{2}}^{*} L^{*} E_{j_{1}}^{*} & \\
& =\sum E_{j_{1}}^{*} L^{*} E_{j_{2}}^{*} L^{*} E_{j_{3}}^{*} L^{*} E_{j_{4}}^{*} L^{*} E_{j_{5}}^{*}
\end{aligned}
$$

where the summations are over all distinct arrangements of $(1,1,1,2,2)$. Define the expressions $\widetilde{K}[1,1,2,2], \widetilde{K}[1,1,2], \ldots$ in a similar way for the tuples $(1,1,2,2),(1,1,2), \ldots$. For any $f \in \mathcal{H}$,

$$
K^{*} f=E_{1}^{*} L^{*} \widetilde{K}[1,1,2,2] f+E_{2}^{*} L^{*} \widetilde{K}[1,1,1,2] f .
$$


Our assumptions imply that $E_{1}^{*}$ and $E_{2}^{*}$ have orthogonal ranges in $\mathcal{K}$, and hence

$$
\begin{aligned}
\left\|K^{*} f\right\|^{2} & =\left\|E_{1}^{*} L^{*} \widetilde{K}[1,1,2,2] f\right\|^{2}+\left\|E_{2}^{*} L^{*} \widetilde{K}[1,1,1,2] f\right\|^{2} \\
\leq & \left\|L^{*}\right\|^{2}\left\{\|\widetilde{K}[1,1,2,2] f\|^{2}+\|\widetilde{K}[1,1,1,2] f\|^{2}\right\} \\
= & \left\|L^{*}\right\|^{2}\left\{\left\|E_{1}^{*} L^{*} \widetilde{K}[1,2,2] f\right\|^{2}+\left\|E_{2}^{*} L^{*} \widetilde{K}[1,1,2] f\right\|^{2}\right. \\
& \left.+\left\|E_{1}^{*} L^{*} \widetilde{K}[1,1,2] f\right\|^{2}+\left\|E_{2}^{*} L^{*} \widetilde{K}[1,1,1] f\right\|^{2}\right\} .
\end{aligned}
$$

By obvious estimates of the separate terms and repetition of the process, we obtain

$$
\begin{aligned}
\left\|K^{*} f\right\|^{2} \leq & 2\left\|L^{*}\right\|^{4}\left\{\|\widetilde{K}[1,2,2] f\|^{2}+\|\widetilde{K}[1,1,2] f\|^{2}+\|\widetilde{K}[1,1,1] f\|^{2}\right\} \\
= & 2\left\|L^{*}\right\|^{4}\left\{\left\|E_{1}^{*} L^{*} \widetilde{K}[2,2] f\right\|^{2}+\left\|E_{2}^{*} L^{*} \widetilde{K}[1,2] f\right\|^{2}\right. \\
& \left.\quad+\left\|E_{1}^{*} L^{*} \widetilde{K}[1,2] f\right\|^{2}+\left\|E_{2}^{*} L^{*} \widetilde{K}[1,1] f\right\|^{2}+\left\|E_{1}^{*} L^{*} \widetilde{K}[1,1] f\right\|^{2}\right\} \\
\leq & 2^{2}\left\|L^{*}\right\|^{6}\left\{\|\widetilde{K}[2,2] f\|^{2}+\|\widetilde{K}[1,2] f\|^{2}+\|\widetilde{K}[1,1] f\|^{2}\right\} \\
= & 2^{2}\left\|L^{*}\right\|^{6}\left\{\left\|E_{2}^{*} L^{*} E_{2}^{*} f\right\|^{2}+\left\|E_{1}^{*} L^{*} E_{2}^{*} f\right\|^{2}\right. \\
& \left.+\left\|E_{2}^{*} L^{*} E_{1}^{*} f\right\|^{2}+\left\|E_{1}^{*} L^{*} E_{1}^{*} f\right\|^{2}\right\} \\
\leq & 2^{3}\left\|L^{*}\right\|^{8}\left\{\left\|E_{2}^{*} f\right\|^{2}+\left\|E_{1}^{*} f\right\|^{2}\right\} \\
= & 2^{4}\left\|L^{*}\right\|^{8}\|f\|^{2} .
\end{aligned}
$$

Hence $\left\|K^{*}\right\| \leq 2^{2}\left\|L^{*}\right\|^{4}$, yielding (41) when $n_{1}=3, n_{2}=2$, and $d=2$.

In the general case, we proceed in a similar way. At the last stage we shall have $\left\|K^{*} f\right\|^{2} \leq d^{n-1}\left\|L^{*}\right\|^{2(n-1)}\|f\|^{2}$, which implies (41).

Theorem 12. Let $\varphi(z)$ be a complex-valued function in the Schur-Agler class on the unit ball. Then for each $z \in \mathbb{B}_{d}$ and any nonnegative integers $n_{1}, \ldots, n_{d}$ which are not all zero,

$$
\left|\frac{\partial^{n} \varphi}{\partial z_{d}^{n_{d}} \cdots \partial z_{1}^{n_{1}}}\right| \leq d^{(n-1) / 2} n_{1} ! n_{2} ! \cdots n_{d} ! \frac{1-|\varphi(z)|^{2}}{\left(1-\|z\|_{2}^{2}\right)\left(1-\|z\|_{2}\right)^{n-1}},
$$

where $n=n_{1}+\cdots+n_{d}$.

This is an improvement on the inequality

$$
\left|\frac{\partial^{n} \varphi}{\partial z_{d}^{n_{d}} \cdots \partial z_{1}^{n_{1}}}\right| \leq n ! \frac{1-|\varphi(z)|^{2}}{\left(1-\|z\|_{2}^{2}\right)\left(1-\|z\|_{2}\right)^{n-1}},
$$

which follows as a corollary of Theorem [10. 
Proof of Theorem 12. When $n=1$, (42) is immediate from (36). Assume $n \geq 2$. Represent $\varphi(z)$ as in (8) with the same choices of Hilbert spaces and operators as in the proof of Theorem 10. Fix $z \in \mathbb{B}_{d}$, and set $L=L(z)$. By (35),

$$
\left|\frac{\partial^{n} \varphi}{\partial z_{d}^{n_{d}} \cdots \partial z_{1}^{n_{1}}}\right| \leq\left\|C\left(I_{\mathcal{H}}-Z(z) A\right)^{-1}\right\|\|K\|\left\|\left(I_{\mathcal{K}}-A Z(z)\right)^{-1} B\right\|,
$$

where $K$ is as in Lemma 11. By (21), (22), and Lemma 11,

$$
\left|\frac{\partial^{n} \varphi}{\partial z_{d}^{n_{d}} \cdots \partial z_{1}^{n_{1}}}\right| \leq \frac{1-|\varphi(z)|^{2}}{1-\|Z(z)\|^{2}} d^{(n-1) / 2}\|L\|^{n-1} .
$$

Hence since $\|L\| \leq 1 /(1-\|Z(z)\|)$ by (23) and $\|Z(z)\|=\|z\|_{2}$ by the proof of Theorem 10, we obtain the inequality (42).

It is not hard to find cases where either one of the inequalities (10) and (42) is better than the other.

\section{Concluding remarks}

Operator-valued functions. The results of 93 and $\$ 4$ extend easily to norm estimates of operator-valued functions which belong to the Schur-Agler class on the polydisk or unit ball. For the definitions of these classes, see Agler 1 and Ball and Trent 11] in the case of the polydisk, and Ball, Trent, and Vinnikov 12 and Eschmeier and Putinar [17] for the unit ball. Operator-valued functions in the Schur-Agler class on the polydisk or unit ball have unitary realizations of the same form as described in $\$ 1.1$ and $\$ 1.2$ for the scalar case. Since such realizations are all that is needed for our main estimates, the results of $\S 3$ and \$4 carry over without essential change to operator-valued functions.

Some open problems. Many questions remain open, for example:

1. The estimates in $\$ 3$ and $\$ 4$ are best possible in one variable [5, 24. The corresponding questions remain to be investigated in general. We note that equality holds at the origin in (28) if $\varphi(z)=z_{1}^{n_{1}} \cdots z_{d}^{n_{d}}$ for some nonnegative integers $n_{1}, \ldots, n_{d}$ which are not all zero. This function belongs to the SchurAgler class on the polydisk by the definition of the class in \$1.1.

2. Are there functions in the Schur class (but not, of course, in the Schur-Agler class) on the polydisk or unit ball that do not satisfy the estimates in 93 and \$4? A well-known example of a function in the Schur class on $\mathbb{D}^{3}$ which is not in the Schur-Agler class on the polydisk is the Kaijser-Varopoulos polynomial [20, 26],

$$
\varphi(z)=\frac{1}{5}\left(z_{1}^{2}+z_{2}^{2}+z_{3}^{2}-2 z_{1} z_{2}-2 z_{1} z_{3}-2 z_{2} z_{3}\right) .
$$

We do not know if this function satisfies the inequalities in $\$ 3$. Examples of functions which belong to the Schur class on the unit ball $\mathbb{B}_{2}$ but are not in the Schur-Agler class on the unit ball are given by Alpay and Kaptanoğlu [3, p. 6]. They have the form

$$
\varphi(z)=z_{1}+c_{1} z_{2}^{2}+c_{2} z_{2}^{4}+\cdots+c_{m} z_{2}^{2 m},
$$


where $m$ is an arbitrary positive integer and $c_{1}, c_{2}, \ldots$ are the coefficients in the expansion $1-\sqrt{1-t}=c_{1} t+c_{2} t^{2}+\cdots$. For each $j \geq 1, c_{j}>0$. We do not know if these functions satisfy the inequalities in 84. Other simple examples for the unit ball are given in [2, pp. 99-100].

3. The Schur-Agler class on the polydisk and unit ball are two concrete classes of analytic functions which admit realizations (3) such that the functions (6) and (7) are linear. Similar ingredients are available in Cartan domains, and this environment may be amenable to analogous treatment. See Upmeier [25] for information on Cartan domains. Realization formulas were first proved for Cartan domains and their products by Ambrozie and Timotin in 4. This was generalized by Ball and Bolotnikov [10] to domains such that $1-Z(z)^{*} Z(z)>0$, where $Z(z)$ is a matrix polynomial.

\section{References}

1. J. Agler, On the representation of certain holomorphic functions defined on a polydisc, Topics in operator theory: Ernst D. Hellinger memorial volume, Oper. Theory Adv. Appl., vol. 48, Birkhäuser, Basel, 1990, pp. 47-66.

2. J. Agler and J. E. McCarthy, Pick interpolation and Hilbert function spaces, Graduate Studies in Mathematics, vol. 44, American Mathematical Society, Providence, RI, 2002.

3. D. Alpay and H. T. Kaptanoğlu, Some finite-dimensional backward-shift-invariant subspaces in the ball and a related interpolation problem, Integral Equations Operator Theory 42 (2002), no. 1, 1-21.

4. C.-G. Ambrozie and D. Timotin, A von Neumann type inequality for certain domains in $\mathbf{C}^{n}$, Proc. Amer. Math. Soc. 131 (2003), no. 3, 859-869.

5. J. M. Anderson and J. Rovnyak, On generalized Schwarz-Pick estimates, Mathematika 53 (2006), 161-168.

6. W. Arveson, Subalgebras of $C^{*}$-algebras. III. Multivariable operator theory, Acta Math. 181 (1998), no. 2, 159-228.

7. F. G. Avkhadiev and K.-J. Wirths, Schwarz-Pick inequalities for derivatives of arbitrary order, Constr. Approx. 19 (2003), no. 2, 265-277.

8. _ Schwarz-Pick inequalities for hyperbolic domains in the extended plane, Geom. Dedicata 106 (2004), 1-10.

9. _ Punishing factors for finitely connected domains, Monatsh. Math. 147 (2006), no. $2,103-115$.

10. J. A. Ball and V. Bolotnikov, Realization and interpolation for Schur-Agler-class functions on domains with matrix polynomial defining function in $\mathbb{C}^{n}$, J. Funct. Anal. 213 (2004), no. $1,45-87$.

11. J. A. Ball and T. T. Trent, Unitary colligations, reproducing kernel Hilbert spaces, and Nevanlinna-Pick interpolation in several variables, J. Funct. Anal. 157 (1998), no. 1, 1-61.

12. J. A. Ball, T. T. Trent, and V. Vinnikov, Interpolation and commutant lifting for multipliers on reproducing kernel Hilbert spaces, Operator theory and analysis (Amsterdam, 1997), Oper. Theory Adv. Appl., vol. 122, Birkhäuser, Basel, 2001, pp. 89-138.

13. C. Bénéteau, A. Dahlner, and D. Khavinson, Remarks on the Bohr phenomenon, Comput. Methods Funct. Theory 4 (2004), no. 1, 1-19.

14. H. Bohr, A theorem concerning power series, Proc. London Math. Soc. (2) 13 (1914), 1-5, Collected Mathematical Works. Vol. III, paper \#E 3, Dansk Matematisk Forening, København, 1952. 
15. M. S. Brodskiŭ, Unitary operator colligations and their characteristic functions, Uspekhi Mat. Nauk 33 (1978), no. 4(202), 141-168, 256, Engl. transl. Russian Math. Surveys 33 (1978), no. 4, 159-191.

16. S. W. Drury, A generalization of von Neumann's inequality to the complex ball, Proc. Amer. Math. Soc. 68 (1978), no. 3, 300-304.

17. J. Eschmeier and M. Putinar, Spherical contractions and interpolation problems on the unit ball, J. Reine Angew. Math. 542 (2002), 219-236.

18. G. E. Knese, A Schwarz lemma on the polydisk, preprint, http://www.citebase.org/ abstract?id=oai : arXiv . org:math/0512452, 2005.

19. E. Landau and D. Gaier, Darstellung und Begründung einiger neuerer Ergebnisse der Funktionentheorie, third ed., Springer-Verlag, Berlin, 1986.

20. B. A. Lotto and T. Steger, von Neumann's inequality for commuting, diagonalizable contractions. II, Proc. Amer. Math. Soc. 120 (1994), no. 3, 897-901.

21. B. D. MacCluer, K. Stroethoff, and R. Zhao, Generalized Schwarz-Pick estimates, Proc. Amer. Math. Soc. 131 (2003), no. 2, 593-599.

22. __ Schwarz-Pick type estimates, Complex Var. Theory Appl. 48 (2003), no. 8, 711730 .

23. W. Rudin, Function theory in polydiscs, W. A. Benjamin, Inc., New York-Amsterdam, 1969.

24. St. Ruscheweyh, Two remarks on bounded analytic functions, Serdica 11 (1985), no. 2, 200-202.

25. H. Upmeier, Toeplitz operators and index theory in several complex variables, Operator Theory: Advances and Applications, vol. 81, Birkhäuser Verlag, Basel, 1996.

26. N. Th. Varopoulos, On an inequality of von Neumann and an application of the metric theory of tensor products to operators theory, J. Functional Analysis 16 (1974), 83-100.

J. Milne Anderson E-MAIL: helen@math.ucl.ac.uk AdDress: Department of Mathematics, University College London, Gower Street, London $W C 1 E$ 6BT, $U K$

Michael A. Dritschel

E-MAIL: m.a.dritschel@ncl.ac.uk ADDRESS: School of Mathematics and Statistics, Merz Court, University of Newcastle upon Tyne, Newcastle upon Tyne NE1 7RU, UK

James Rovnyak

E-MAIL: rovnyak@virginia.edu AdDress: Department of Mathematics, University of Virginia, P. O. Box 400137, Charlottesville, VA 22904, USA 